\section{Getting back to practice}

Give your patients confidence that your clinic is safe and protect yourself and your associates.

As a dentist, you need to get back to work as quickly as possible, while making sure that you, your team and your patients are well protected.

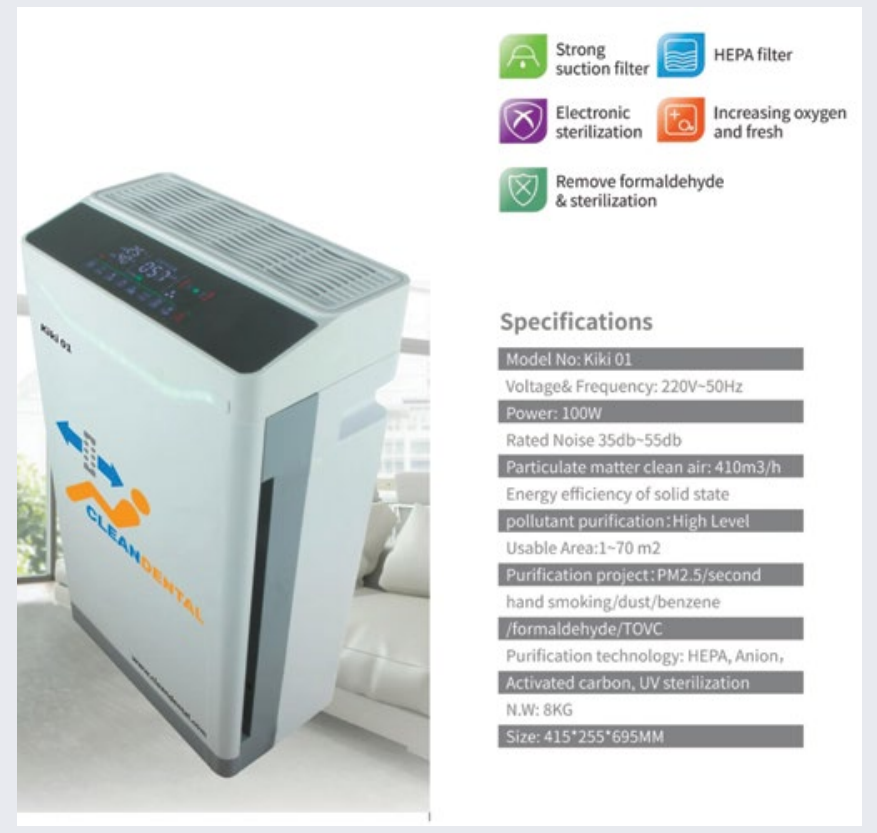

Essential will be clean rooms and the reduction of 'risk'. This can be achieved using:

- Air filtration in the clinic, waiting room and reception

- Detection of patients with a temperature using a thermal camera at reception

- External high-volume aspiration units to remove $99.9 \%$ of dental aerosols

- Reception perspex screens

- Appropriate PPE.

Further information can be found on newly launched www. cleandental.co.uk which will cover:

- COVID-19 - What happens in the lungs?

- Clear guidance on PPE

- How to 'pre-screen' patients

- How to manage reception

- How to control patient flow in a practice

- The use of the waiting room

- The clinical room

- Decontamination and infection control protocols

- The anticipated new 'normal' post COVID-19

- The stages expected as we progress through the stages of risk and 'immunity'.

For information email info@cleandental.co.uk.

\section{Remove the headache}

The first tax return of the year might be out of the way but with another one looming some of you might be wondering if there's a way to remove the headache and make the process easier.

You'll be pleased to know that there is - give the responsibility to accountants4dentists.

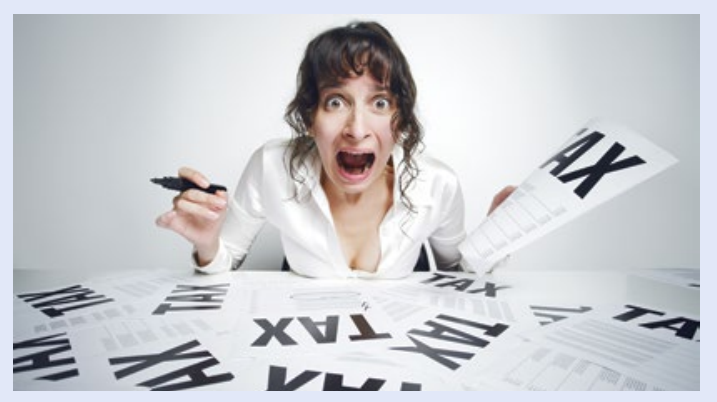

Highly experienced and renowned for providing a first-class professional service to dentists, the team is well equipped to take care of all your accounts preparation and tax compliance, giving you one less thing to worry about. Should you desire, an accountant can even take care of your bookkeeping and help you plan for the year ahead.

To find out more how the team could help with your upcoming tax return and beyond, contact accountants4dentists today.

For more information call 08453455060 or 0754 DENTIST.

Email info@4dentistsgroup.com or visit www.4dentistsgroup.com.

\section{Gentle and effective}

CS Toothbrushes from Curaprox are made using Curen filaments, rather than nylon, which makes them gentle yet still incredibly effective. Curaprox is able to pack thousands - as opposed to the hundreds on a standard manual toothbrush - of these filaments on the brush head, which ensures unparalleled cleaning.

Not only that, the small, compact head allows users to reach all areas of the mouth, including those ever-neglected upper molars. The octagonal handle allows a comfortable grip that helps users to position the brush at the correct angle, ensuring the gum line is cleaned effectively.

Make sure you are armed with helpful tools and advice to keep your patients' oral hygiene regime on top form. Contact the friendly team at Curaprox today to discover more about the gentle and effective CS Toothbrushes.

For more information call 01480 862084, email info@curaprox. co.uk or visit www.curaprox.co.uk.

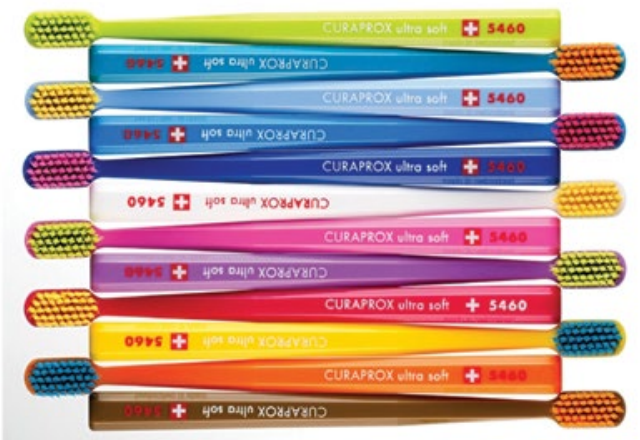

